\title{
Re-embedding a 1-Plane Graph into a Straight-line Drawing in Linear Time *
}

\author{
Seok-Hee Hong ${ }^{1}$ and Hiroshi Nagamochi ${ }^{2}$ \\ 1 University of Sydney, Australia \\ seokhee.hong@sydney.edu.au \\ 2 Kyoto University, Japan \\ nag@amp.i.kyoto-u.ac.jp
}

\begin{abstract}
Thomassen characterized some 1-plane embedding as the forbidden configuration such that a given 1-plane embedding of a graph is drawable in straight-lines if and only if it does not contain the configuration [C. Thomassen, Rectilinear drawings of graphs, J. Graph Theory, 10(3), 335-341, 1988].

In this paper, we characterize some 1-plane embedding as the forbidden configuration such that a given 1-plane embedding of a graph can be reembedded into a straight-line drawable 1-plane embedding of the same graph if and only if it does not contain the configuration. Re-embedding of a 1-plane embedding preserves the same set of pairs of crossing edges. We give a linear-time algorithm for finding a straight-line drawable 1plane re-embedding or the forbidden configuration.
\end{abstract}

\section{Introduction}

Since the 1930s, a number of researchers have investigated planar graphs. In particular, a beautiful and classical result, known as Fáry's Theorem, asserts that every plane graph admits a straight-line drawing [5]. Indeed, a straight-line drawing is the most popular drawing convention in Graph Drawing.

More recently, researchers have investigated 1-planar graphs (i.e., graphs that can be embedded in the plane with at most one crossing per edge), introduced by Ringel [13]. Subsequently, the structure of 1-planar graphs has been investigated $[4,12]$. In particular, Pach and Toth [12] proved that a 1-planar graph with $n$ vertices has at most $4 n-8$ edges, which is a tight upper bound. Unfortunately, testing the 1-planarity of a graph is NP-complete [6,11], however linear-time algorithms are available for special subclasses of 1-planar graphs $[1$, $3,7]$.

Thomassen [14] proved that every 1-plane graph (i.e., a 1-planar graph embedded with a given 1-plane embedding) admits a straight-line drawing if and only if it does not contain any of two special 1-plane graphs, called the $B$ configuration or $W$-configuration, see Fig. 1.

\footnotetext{
* Research supported by ARC Future Fellowship and ARC Discovery Project DP160104148. This is an extended abstract. For a full version with omitted proofs, see [9].
} 


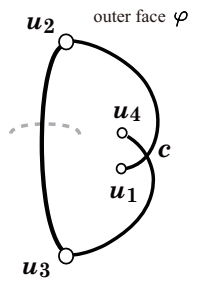

(a)

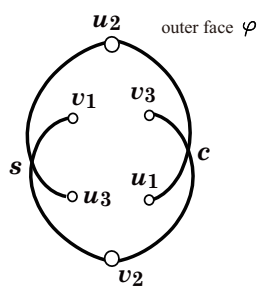

(b)

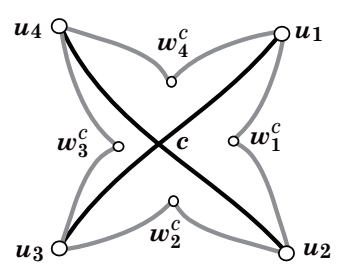

(c)

Fig. 1. (a) B-configuration with three edges $u_{1} u_{2}, u_{2} u_{3}$ and $u_{3} u_{4}$ and one crossing $c$ made by an edge pair $\left\{u_{1} u_{2}, u_{3} u_{4}\right\}$, where edge $u_{2} u_{3}$ may have a crossing when the configuration is part of a 1-plane embedding; (b) W-configuration with four edges $u_{1} u_{2}$, $u_{2} u_{3}, v_{1} v_{2}$ and $v_{2} v_{3}$ and two crossings $c$ and $s$ made by edge pairs $\left\{u_{1} u_{2}, v_{2} v_{3}\right\}$ and $\left\{u_{2} u_{3}, v_{1} v_{2}\right\}$, where possibly $u_{1}=v_{1}$ and $u_{3}=v_{3}$; (c) Augmenting a crossing $c \in \chi$ made by edges $u_{1} u_{3}$ and $u_{2} u_{4}$ with a new cycle $Q_{c}=\left(u_{1}, w_{1}^{c}, u_{2}, w_{2}^{c}, u_{3}, w_{3}^{c}, u_{4}, w_{4}^{c}\right)$ depicted by gray lines.

Recently, Hong et al. [8] gave an alternative constructive proof, with a lineartime testing algorithm and a drawing algorithm. They also showed that some 1-planar graphs need an exponential area with straight-line drawing.

We call a 1-plane embedding straight-line drawable (SLD for short) if it admits a straight-line drawing, i.e., it does not contain a B- or W-configuration by Thomassen [14]. In this paper, we investigate a problem of "re-embedding" a given non-SLD 1-plane embedding $\gamma$ into an SLD 1-plane embedding $\gamma^{\prime}$. For a given 1-plane embedding $\gamma$ of a graph $G$, we call another 1-plane embedding $\gamma^{\prime}$ of $G$ a cross-preserving embedding of $\gamma$ if exactly the same set of edge pairs make the same crossings in $\gamma^{\prime}$.

More specifically, we first characterize the forbidden configuration of 1-plane embeddings that cannot admit an SLD cross-preserving 1-plane embedding. Based on the characterization, we present a linear-time algorithm that either detects the forbidden configuration in $\gamma$ or computes an SLD cross-preserving 1-plane embedding $\gamma^{\prime}$.

Formally, the main problem considered in this paper is defined as follows.

\section{Re-embedding a 1-Plane Graph into a Straight-line Drawing}

Input: A 1-planar graph $G$ and a 1-plane embedding $\gamma$ of $G$.

Output: Test whether $\gamma$ admits an SLD cross-preserving 1-plane embedding $\gamma^{\prime}$, and construct such an embedding $\gamma^{\prime}$ if one exists, or report the forbidden configuration.

To design a linear-time implementation of our algorithm in this paper, we introduce a rooted-forest representation of non-intersecting cycles and an efficient procedure of flipping subgraphs in a plane graph. Since these data structure and procedure can be easily implemented, it has advantage over the complicated decomposition of biconnected graphs into triconnected components [10] or the SPQR tree [2]. 


\section{Plane Embeddings and Inclusion Forests}

Let $U$ be a set of $n$ elements, and let $\mathcal{S}$ be a family of subsets $S \subseteq U$. We say that two subsets $S, S^{\prime} \subseteq U$ are intersecting if none of $S \cap S^{\prime}, S-S^{\prime}$ and $S^{\prime}-S$ is empty. We call $\mathcal{S}$ a laminar if no two subsets in $\mathcal{S}$ are intersecting. For a laminar $\mathcal{S}$, the inclusion-forest of $\mathcal{S}$ is defined to be a forest $\mathcal{I}=(\mathcal{S}, \mathcal{E})$ of a disjoint union of rooted trees such that (i) the sets in $\mathcal{S}$ are regarded as the vertices of $\mathcal{I}$, and (ii) a set $S$ is an ancestor of a set $S^{\prime}$ in $\mathcal{I}$ if and only if $S^{\prime} \subseteq S$.

Lemma 1. For a cyclic sequence $\left(u_{1}, u_{2}, \ldots, u_{\delta}\right)$ of $\delta \geq 2$ elements, define an interval $(i, j)$ to be the set of elements $u_{k}$ with $i \leq k \leq j$ if $i \leq j$ and $(i, j)=$ $(i, \delta) \cup(1, j)$ if $i>j$. Let $\mathcal{S}$ be a set of intervals. A pair of two intersecting intervals in $\mathcal{S}$ (when $\mathcal{S}$ is not a laminar) or the inclusion-forest of $\mathcal{S}$ (when $\mathcal{S}$ is a laminar $)$ can be obtained in $O(\delta+|\mathcal{S}|)$ time.

Throughout the paper, a graph $G=(V, E)$ stands for a simple undirected graph. The set of vertices and the set of edges of a graph $G$ are denoted by $V(G)$ and $E(G)$, respectively. For a vertex $v$, let $E(v)$ be the set of edges incident to $v, N(v)$ be the set of neighbors of $v$, and $\operatorname{deg}(v)$ denote the degree $|N(v)|$ of $v$. A simple path with end vertices $u$ and $v$ is called a $u, v$-path. For a subset $X \subseteq V$, let $G-X$ denote the graph obtained from $G$ by removing the vertices in $X$ together with the edges in $\cup_{v \in X} E(v)$.

A drawing $D$ of a graph $G$ is a geometric representation of the graph in the plane, such that each vertex of $G$ is mapped to a point in the plane, and each edge of $G$ is drawn as a curve. A drawing $D$ of a graph $G=(V, E)$ is called planar if there is no edge crossing. A planar drawing $D$ of a graph $G$ divides the plane into several connected regions, called faces, where a face enclosed by a closed walk of the graph is called an inner face and the face not enclosed by any closed walk is called the outer face.

A planar drawing $D$ induces a plane embedding $\gamma$ of $G$, which is defined to be a pair $(\rho, \varphi)$ of the rotation system (i.e., the circular ordering of edges for each vertex) $\rho$, and the outer face $\varphi$ whose facial cycle $C_{\varphi}$ gives the outer boundary of $D$. Let $\gamma=(\rho, \varphi)$ be a plane embedding of a graph $G=(V, E)$. We denote by $F(\gamma)$ the set of faces in $\gamma$, and by $C_{f}$ the facial cycle determined by a face $f \in F$, where we call a subpath of $C_{f}$ a boundary path of $f$. For a simple cycle $C$ of $G$, the plane is divided by $C$ in two regions, one containing only inner faces and the other containing the outer area, where we say that the former is enclosed by $C$ or the interior of $C$, while the latter is called the exterior of $C$. We denote by $F_{\text {in }}(C)$ the set of inner faces in the interior of $C$, by $E_{\text {in }}(C)$ the set of edges in $E\left(C_{f}\right)$ with $f \in F_{\text {in }}(C)$, and by $V_{\text {in }}(C)$ the set of end-vertices of edges in $E_{\text {in }}(C)$. Analogously define $F_{\mathrm{ex}}(C), E_{\mathrm{ex}}(C)$ and $V_{\mathrm{ex}}(C)$ in the exterior of $C$. Note that $E(C)=E_{\text {in }}(C) \cap E_{\text {ex }}(C)$ and $V(C)=V_{\text {in }}(C) \cap V_{\text {ex }}(C)$.

For a subgraph $H$ of $G$, we define the embedding $\left.\gamma\right|_{H}$ of $\gamma$ induced by $H$ to be a sub-embedding of $\gamma$ obtained by removing the vertices/edges not in $H$, keeping the same rotation system around each of the remaining vertices/crossings and the same outer face. 


\subsection{Inclusion Forests of Inclusive Set of Cycles}

In this and next subsections, let $(G, \gamma)$ stand for a plane embedding of $\gamma=(\rho, \varphi)$ of a biconnected simple graph $G=(V, E)$ with $n=|V| \geq 3$.

Let $C$ be a simple cycle in $G$. We define the direction of $C$ to be an ordered pair $(u, v)$ with $u v \in E(C)$ such that the inner faces in $F_{\text {in }}(C)$ appear on the right hand side when we traverse $C$ in the order that we start $u$ and next visit $v$. For simplicity, we say that two simple cycles $C$ and $C^{\prime}$ are intersecting if $F_{\text {in }}(C)$ and $F_{\text {in }}\left(C^{\prime}\right)$ are intersecting.

Let $\mathcal{C}$ be a set of simple cycles in $G$. We call $\mathcal{C}$ inclusive if no two cycles in $\mathcal{C}$ are intersecting, i.e., $\left\{F_{\text {in }}(C) \mid C \in \mathcal{C}\right\}$ is a laminar. When $\mathcal{C}$ is inclusive, the inclusion-forest of $\mathcal{C}$ is defined to be a forest $\mathcal{I}=(\mathcal{C}, \mathcal{E})$ of a disjoint union of rooted trees such that:

(i) the cycles in $\mathcal{C}$ are regarded as the vertices of $\mathcal{I}$, and

(ii) a cycle $C$ is an ancestor of a cycle $C^{\prime}$ in $\mathcal{I}$ if and only if $F_{\text {in }}\left(C^{\prime}\right) \subseteq F_{\text {in }}(C)$.

Let $\mathcal{I}(\mathcal{C})$ denote the inclusion-forest of $\mathcal{C}$. For a vertex subset $X \subseteq V$, let $\mathcal{C}(X)$ denote the set of cycles $C \in \mathcal{C}$ such that $x \in V(C)$ for some vertex $x \in X$, where we denote $\mathcal{C}(\{v\})$ by $\mathcal{C}(v)$ for short.

Lemma 2. For $(G, \gamma)$, let $\mathcal{C}$ be a set of simple cycles of $G$. Then any of the following tasks can be executed in $O\left(n+\sum_{C \in \mathcal{C}}|E(C)|\right)$ time.

(i) Decision of the directions of all cycles in $\mathcal{C}$;

(ii) Detection of a pair of two intersecting cycles in $\mathcal{C}$ when $\mathcal{C}$ is not inclusive, and construction of the inclusion-forests $\mathcal{I}(\mathcal{C}(v))$ for all vertices $v \in V$ when $\mathcal{C}$ is inclusive; and

(iii) Construction of the inclusion-forest $\mathcal{I}(\mathcal{C})$ when $\mathcal{C}$ is inclusive.

\section{$2.2 \quad$ Flipping Spindles}

A simple cycle $C$ of $G$ is called a spindle (or a $u, v$-spindle) of $\gamma$ if there are two vertices $u, v \in V(C)$ such that no vertex in $V(C)-\{u, v\}$ is adjacent to any vertex in the exterior of $C$, where we call vertices $u$ and $v$ the junctions of $C$. Note that each of the two subpaths of $C$ between $u$ and $v$ is a boundary path of some face in $F(\gamma)$.

Given $(G, \gamma)$, we denote the rotation system around a vertex $v \in V$ by $\rho_{\gamma}(v)$. For a spindle $C$ in $\gamma$, let $J(C)$ denote the set of the two junctions of $C$.

Flipping a u,v-spindle $C$ means to modify the rotation system of vertices in $V_{\text {in }}(C)$ as follows:

(i) For each vertex $w \in V_{\text {in }}(C)-J(C)$, reverse the cyclic order of $\rho_{\gamma}(w)$; and

(ii) For each vertex $u \in J(C)$, reverse the order of subsequence of $\rho_{\gamma}(u)$ that consists of vertices $N(u) \cap V_{\text {in }}(C)$.

Every two distinct spindles $C$ and $C^{\prime}$ in $\gamma$ are non-intersecting, and they always satisfy one of $E_{\text {in }}(C) \cap E_{\text {in }}\left(C^{\prime}\right)=\emptyset, E_{\text {in }}(C) \subseteq E_{\text {in }}\left(C^{\prime}\right)$, and $E_{\text {in }}\left(C^{\prime}\right) \subseteq$ $E_{\text {in }}(C)$. Let $\mathcal{C}$ be a set of spindles in $\gamma$, which is always inclusive, and let $\mathcal{I}(\mathcal{C})$ denote the inclusion-forest of $\mathcal{C}$. 
When we modify the current embedding $\gamma$ by flipping each spindle in $\mathcal{C}$, the resulting embedding $\gamma_{\mathcal{C}}$ is the same, independent from the ordering of the flipping operation to the spindles, since for two spindles $C$ and $C^{\prime}$ which share a common junction vertex $u \in J(C) \cap J\left(C^{\prime}\right)$, the sets $N(u) \cap V_{\text {in }}(C)$ and $N(u) \cap V_{\text {in }}\left(C^{\prime}\right)$ do not intersect, i.e., they are disjoint or one is contained in the other.

Define the depth of a vertex $v \in V$ in $\mathcal{I}$ to be the number of spindles $C \in \mathcal{C}$ such that $v \in V_{\text {in }}(C)-J(C)$, and denote by $\mathrm{p}(v)$ the parity of depth of vertex $v$, i.e., $\mathrm{p}(v)=1$ if the depth is odd and $\mathrm{p}(v)=-1$ otherwise.

For a vertex $v \in V$, let $\mathcal{C}[v]$ denote the set of spindles $C \in \mathcal{C}$ such that $v \in J(C)$, and let $\gamma_{\mathcal{C}[v]}$ be the embedding obtained from $\gamma$ by flipping all spindles in $\mathcal{C}[v]$. Let $\operatorname{rev}\langle\sigma\rangle$ mean the reverse of a sequence $\sigma$. Then we see that $\rho_{\gamma_{\mathcal{C}}}(v)=\rho_{\gamma_{\mathcal{C}[v]}}(v)$ if $\mathrm{p}(v)=1$; and $\rho_{\gamma_{\mathcal{C}}}(v)=\operatorname{rev}\left\langle\rho_{\gamma_{\mathcal{C}[v]}}(v)\right\rangle$ otherwise. To obtain the embedding $\gamma_{\mathcal{C}}$ from the current embedding $\gamma$ by flipping each spindle in $\mathcal{C}$, it suffices to show how to compute each of $\mathrm{p}(v)$ and $\rho_{\gamma_{\mathcal{C}[v]}}(v)$ for all vertices $v \in V$.

Lemma 3. Given $(G, \gamma)$, let $\mathcal{C}$ be a set of spindles of $\gamma$. Then any of the following tasks can be executed in $O\left(n+\sum_{C \in \mathcal{C}}|E(C)|\right)$ time.

(i) Decision of parity $\mathrm{p}(v)$ of all vertices $v \in V$; and

(ii) Computation of $\rho_{\gamma_{\mathcal{C}[v]}}(v)$ for all vertices $v \in V$.

\section{Re-embedding 1-plane Graph and Forbidden Configuration}

A drawing $D$ of a graph $G=(V, E)$ is called a 1-planar drawing if each edge has at most one crossing. A 1-planar drawing $D$ of graph $G$ induces a 1-plane embedding $\gamma$ of $G$, which is defined to be a tuple $(\chi, \rho, \varphi)$ of the crossing system $\chi$ of $E$, the rotation system $\rho$ of $V$, and the outer face $\varphi$ of $D$. The planarization $\mathcal{G}(G, \gamma)$ of a 1-plane embedding $\gamma$ of graph $G$ is the plane embedding obtained from $\gamma$ by regarding crossings also as graph vertices, called crossing-vertices. The set of vertices in $\mathcal{G}(G, \gamma)$ is given by $V \cup \chi$. For a notational convenience, we refer to a subgraph/face of $\mathcal{G}(G, \gamma)$ as a subgraph/face in $\gamma$.

Let $\gamma=(\chi, \rho, \varphi)$ be a 1-plane embedding of graph $G$. We call another 1-plane embedding $\gamma^{\prime}=\left(\chi^{\prime}, \rho^{\prime}, \varphi^{\prime}\right)$ of graph $G$ a cross-preserving 1-plane embedding of $\gamma$ when the same set of edge pairs makes crossings, i.e., $\chi=\chi^{\prime}$. In other words, the planarization $\mathcal{G}\left(G, \gamma^{\prime}\right)$ is another plane embedding of $\mathcal{G}(G, \gamma)$ such that the alternating order of edges incident to each crossing-vertex $c \in \chi$ is preserved.

To eliminate the additional constraint on the rotation system on each crossingvertex $c \in \chi$, we introduce "circular instances." We call an instance $(G, \gamma)$ of 1-plane embedding circular when for each crossing $c \in \chi$, the four end-vertices of the two crossing edges $u_{1} u_{3}$ and $u_{2} u_{4}$ that create $c$ (where $u_{1}, u_{2}, u_{3}$ and $u_{4}$ appear in the clockwise order around $c$ ) are contained in a cycle $Q_{c}=$ $\left(u_{1}, w_{1}^{c}, u_{2}, w_{2}^{c}, u_{3}, w_{3}^{c}, u_{4}, w_{4}^{c}\right)$ of eight crossing-free edges for some vertices $w_{i}^{c}$, $i=1,2,3,4$ of degree 2, as shown in Fig. 1(c). By definition, $c$ and each $w_{i}^{c}$ not necessarily appear along the same facial cycle in the planarization $\mathcal{G}(G, \gamma)$. For example, path $(v, w, u)$ is part of such a cycle $Q_{s}$ for the crossing $s$ in the 
circular instance in Fig. 2(a), but $c$ and $w$ are not on the same facial cycle in the planarization.

A given instance can be easily converted into a circular instance by augmenting the end-vertices of each pair of crossing edges as follows. In the plane graph, $\mathcal{G}(G, \gamma)$, for each crossing-vertex $c \in \chi$ and its neighbors $u_{1}, u_{2}, u_{3}$ and $u_{4}$ that appear in the clockwise order around $c$, we add a new vertex $w_{i}^{c}, i=1,2,3,4$ and eight new edges $u_{i} w_{i}^{c}$ and $w_{i}^{c} u_{i+1}, i=1,2,3,4$ (where $u_{5}$ means $u_{1}$ ) to form a cycle $Q_{c}$ of length 8 whose interior contains no other vertex than $c$.

Let $H$ be the resulting graph augmented from $G$, and let $\Gamma$ be the resulting 1-plane embedding of $H$ augmented from $\gamma$. Note that $|V(H)| \leq|V(G)|+4|\chi|$ holds. We easily see that if $\gamma$ admits an SLD cross-preserving embedding $\gamma^{\prime}$ then $\Gamma$ admits an SLD cross-preserving embedding $\Gamma^{\prime}$. This is because a straight-line drawing $D_{\gamma^{\prime}}$ of $\gamma^{\prime}$ can be changed into a straight-line drawing $D_{\Gamma^{\prime}}$ of some crosspreserving embedding $\Gamma^{\prime}$ of $\Gamma$ by placing the newly introduced vertices $w_{i}^{c}$ within the region sufficiently close to the position of $c$. We here see that cycle $Q_{c}$ can be drawn by straight-line segments without intersecting with other straight-line segments in $D_{\gamma^{\prime}}$.

Note that the instance $\left(G, \gamma^{\prime}\right)$ remains circular for any cross-preserving embedding $\gamma^{\prime}$ of $\gamma$. In the rest of paper, let $(G, \gamma)$ stand for a circular instance $(G=(V, E), \gamma=(\chi, \rho, \varphi))$ with $n \geq 3$ vertices and let $\mathcal{G}$ denote its planarization $\mathcal{G}(G, \gamma)$. Fig. 2 shows examples of circular instances $(G, \gamma)$, where the vertexconnectivity of $\mathcal{G}$ is 1 .

As an important property of a circular instance, the subgraph $G_{(0)}$ with crossing-free edges is a spanning subgraph of $G$ and the four end-vertices of any two crossing edges are contained in the same block of the graph $G_{(0)}$. The biconnectivity is necessary to detect certain types of cycles by applying Lemma 2 .

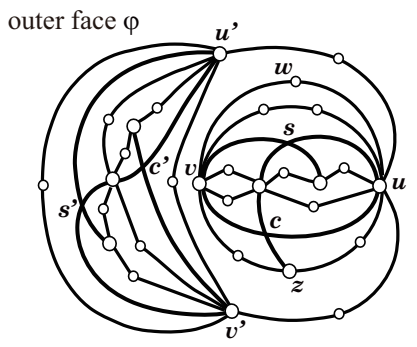

(a)

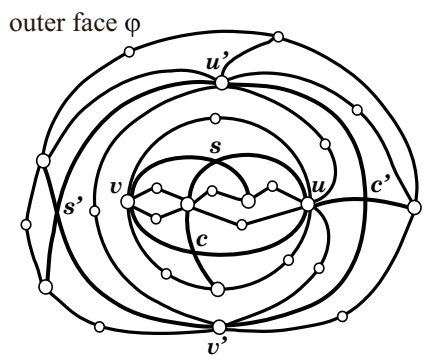

(b)

Fig. 2. Circular instances $(G, \gamma)$ with a cut-vertex $u$ of $\mathcal{G}$, where the crossing edges are depicted by slightly thicker lines: (a) hard B-cycles $C=(u, c, v, s)$ and $C^{\prime}=$ $\left(u^{\prime}, c^{\prime}, v^{\prime}, s^{\prime}\right)$, (b) hard B-cycle $C=(u, c, v, s)$ and a nega-cycle $C^{\prime}=\left(u^{\prime}, c^{\prime}, v^{\prime}, s^{\prime}\right)$ whose reversal is a hard B-cycle, where vertices $u, v, u^{\prime}, v^{\prime} \in V$ and crossings $c, s, c^{\prime}, s^{\prime} \in \chi$. 


\subsection{Candidate Cycles, B/W Cycle, Posi/Nega Cycle, Hard/Soft Cycle}

For a circular instance $(G, \gamma)$, finding a cross-preserving embedding of $\gamma$ is effectively equivalent to finding another plane embedding of $\mathcal{G}$ so that all the current $\mathrm{B}$ - and W-configurations are eliminated and no new B- or W-configurations are introduced. To detect the cycles that can be the boundary of a B- or Wconfiguration in changing the plane embedding of $\mathcal{G}$, we categorize cycles containing crossing vertices in $\mathcal{G}$.

A candidate posi-cycle (resp., candidate nega-cycle) in $\mathcal{G}$ is defined to be a cycle $C=(u, c, v)$ or $C=(u, c, v, s)$ in $\mathcal{G}$ with $u, v \in V$ and $c, s \in \chi$ such that the interior (resp., exterior) of $C$ does not contain a crossing-free edge $u v \in E$ and any other crossing vertex $c^{\prime}$ adjacent to both $u$ and $v$.

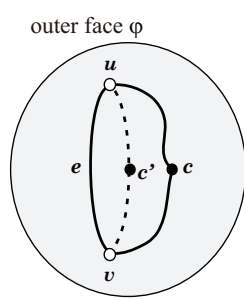

(a) $C \in C^{\mathrm{p}}$

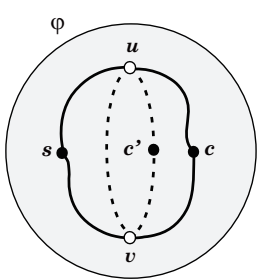

(b) $C \in C^{\mathrm{p}}$

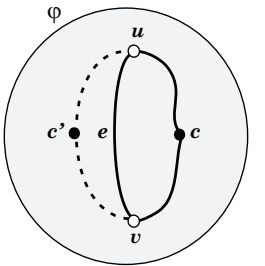

(c) $C \in C^{\mathrm{n}}$

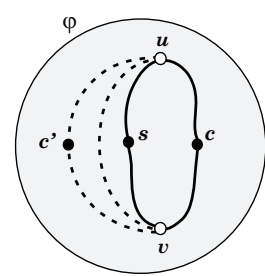

(d) $C \equiv C^{\mathrm{n}}$

Fig. 3. Candidate posi- and nega-cycles $C=(u, c, v)$ and $C=(u, c, v, s)$ in $\mathcal{G}$, where white circles represent vertices in $V$ while black ones represent crossings in $\chi$ : (a) candidate posi-cycle of length 3 , (b) candidate posi-cycle of length 4 , (c) candidate nega-cycle of length 3 , and (d) candidate nega-cycle of length 4 .

Fig. 3(a)-(b) and (c)-(d) illustrate candidate posi-cycles and candidate negacycles, respectively. Let $\mathcal{C}^{\mathrm{p}}$ and $\mathcal{C}^{\mathrm{n}}$ be the sets of candidate posi-cycles and candidate nega-cycles, respectively. By definition we see that the set $\mathcal{C}^{\mathrm{p}} \cup \mathcal{C}^{\mathrm{n}} \cup\left\{C_{f} \mid\right.$ $f \in F(\gamma)\}$ is inclusive, and hence $\left|\mathcal{C}^{\mathrm{p}} \cup \mathcal{C}^{\mathrm{n}} \cup\left\{C_{f} \mid f \in F(\gamma)\right\}\right|=O(n)$.

A candidate posi-cycle $C$ with $C=(u, c, v)$ (resp., $C=(u, c, v, s))$ is called a B-cycle if

(a)-(B): the exterior of $C$ contains no vertices in $V-\{u, v\}$ adjacent to $c$ (resp., contains exactly one vertex in $V-\{u, v\}$ adjacent to $c$ or $s$ ).

Note that $u v \in E$ when $C=(u, c, v)$ is a B-cycle, as shown in Fig. 4(a). Fig. 4(b) and (d) illustrate the other types of B-cycles.

A candidate posi-cycle $C=(u, c, v, s)$ is called a $W$-cycle if

(a)-(W): the exterior of $C$ contains no vertices in $V-\{u, v\}$ adjacent to $c$ or $s$.

Fig. 4(c) and (e) illustrate W-cycles.

Let $\mathcal{C}_{\mathrm{W}}$ (resp., $\mathcal{C}_{\mathrm{B}}$ ) be the set of $\mathrm{W}$-cycles (resp., B-cycles) in $\gamma$. Clearly a $\mathrm{W}$-cycle (resp., B-cycle) gives rise to a $\mathrm{W}$-configuration (resp., B-configuration). 


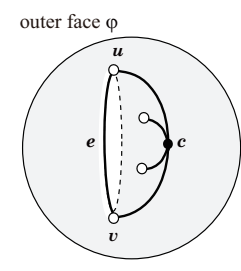

(a) $C \in C_{\mathrm{B}}-C^{+}$

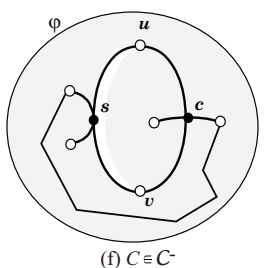

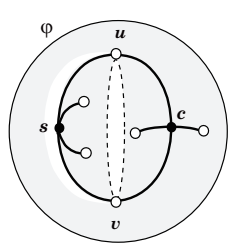

(b) $C \equiv C_{\mathrm{B}}-C^{+}$

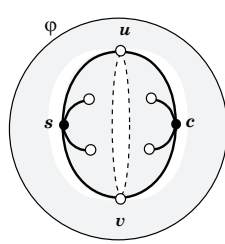

(c) $C \in \mathrm{CW}_{\mathrm{W}}-\mathrm{C}^{+}$

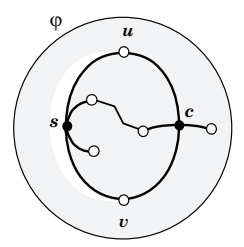

(d) $C \in C_{\mathrm{B}} \cap C^{+}$

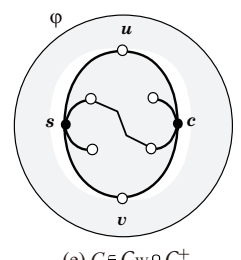

(e) $C \in C_{W} \cap C^{+}$
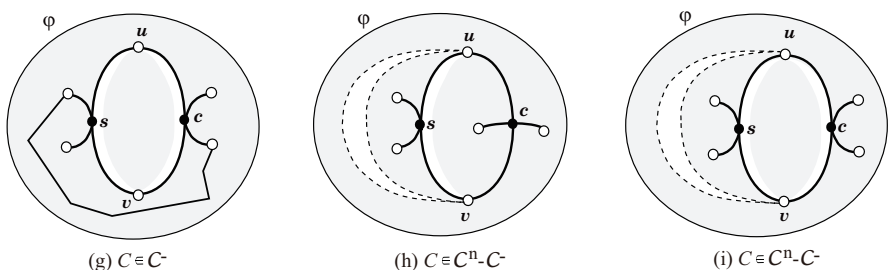

Fig. 4. Illustration of types of cycles $C=(u, c, v)$ and $C=(u, c, v, s)$ in $\mathcal{G}$, where white circles represent vertices in $V$ while black ones represent crossings in $\chi$ : (a) B-cycle of length 3, which is always soft, (b) soft B-cycle of length 4, (c) soft W-cycle, (d) hard B-cycle of length 4, (e) hard W-cycle, (f) nega-cycle whose reversal is a hard B-cycle, (g) nega-cycle whose reversal is a hard $\mathrm{W}$-cycle, (h) candidate nega-cycle of length 4 that is not a nega-cycle whose reversal is a hard B-cycle, and (i) candidate nega-cycle of length 4 that is not a nega-cycle whose reversal is a hard W-cycle.

Conversely, by choosing a W-configuration (resp., B-configuration) so that the interior is minimal, we obtain a W-cycle (resp., B-cycle). Hence we observe that the current embedding $\gamma$ admits a straight-line drawing if and only if $\mathcal{C}_{\mathrm{W}}=\mathcal{C}_{\mathrm{B}}=$ $\emptyset$.

A W- or B-cycle $C$ is called hard if

(b): length of $C$ is 4 , and the interior of $C=(u, c, v, s)$ contains no inner face $f$ whose facial cycle $C_{f}$ contains both vertices $u$ and $v$, i.e., some path connects $c$ and $s$ without passing through $u$ or $v$.

On the other hand, a $\mathrm{W}$ - or B-cycle $C=(u, c, v, s)$ of length 4 that does not satisfy condition (b) or a B-cycle of length 3 is called soft. We also call a hard $\mathrm{B}$ - or W-cycle a posi-cycle.

Fig. 4(d) and (e) illustrate a hard B-cycle and a hard W-cycles, respectively, whereas Fig. 4(a) and (b) (resp., (c)) illustrate soft B-cycles (resp., a soft Wcycle).

A cycle $C=(u, c, v, s)$ is called a nega-cycle if it becomes a posi-cycle when an inner face in the interior of $C$ is chosen as the outer face. In other words, a nega-cycle is a candidate nega-cycle $C=(u, c, v, s)$ of length 4 that satisfies the following conditions (a') and (b'), where (a') (resp., (b')) is obtained from the above conditions (a)-(B) and (a)-(W) (resp., (b)) by exchanging the roles of "interior" and "exterior":

(a'): the interior of $C$ contains at most one vertex in $V-\{u, v\}$ adjacent to $c$ or $s$; and 
(b'): the exterior of $C$ contains no face $f$ whose facial cycle $C_{f}$ contains both vertices $u$ and $v$.

Fig. 4(f) and (g) illustrate nega-cycles, whereas Fig. 4(h) and (i) illustrate candidate nega-cycles that are not nega-cycles.

Let $\mathcal{C}^{+}$(resp., $\mathcal{C}^{-}$) denote the set of posi-cycles (resp., nega-cycles) in $\gamma$. By definition, it holds that $\mathcal{C}^{+} \subseteq \mathcal{C}_{\mathrm{W}} \cup \mathcal{C}_{\mathrm{B}} \subseteq \mathcal{C}^{\mathrm{p}}$ and $\mathcal{C}^{-} \subseteq \mathcal{C}^{\mathrm{n}}$.

\subsection{Forbidden Cycle Pairs}

We define a forbidden configuration that characterizes 1-plane embeddings, which cannot be re-embedded into SLD ones. A forbidden cycle pair is defined to be a pair $\left\{C, C^{\prime}\right\}$ of a posi-cycle $C=(u, c, v, s)$ and a posi- or nega-cycle $C^{\prime}=\left(u^{\prime}, c^{\prime}, v^{\prime}, s^{\prime}\right)$ in $\mathcal{G}$ with $u, v, u^{\prime}, v^{\prime} \in V$ and $c, s, c^{\prime}, s^{\prime} \in \chi$ to which $\mathcal{G}$ has a $u, u^{\prime}$-path $P_{1}$ and a $v, v^{\prime}$-path $P_{2}$ such that:

(i) when $C^{\prime} \in \mathcal{C}^{+}$, paths $P_{1}$ and $P_{2}$ are in the exterior of $C$ and $C^{\prime}$, i.e., $V\left(P_{1}\right)$ $\left\{u, u^{\prime}\right\}, V\left(P_{2}\right)-\left\{v, v^{\prime}\right\} \subseteq V_{\mathrm{ex}}(C) \cap V_{\mathrm{ex}}\left(C^{\prime}\right)$, where $C$ and $C^{\prime}$ cannot have any common inner face; and

(ii) when $C^{\prime} \in \mathcal{C}^{-}$, paths $P_{1}$ and $P_{2}$ are in the exterior of $C$ and the interior of $C^{\prime}$, i.e., $V\left(P_{1}\right)-\left\{u, u^{\prime}\right\}, V\left(P_{2}\right)-\left\{v, v^{\prime}\right\} \subseteq V_{\text {ex }}(C) \cap V_{\text {in }}\left(C^{\prime}\right)$, where $C$ is enclosed by $C^{\prime}$.

In (i) and (ii), $P_{1}$ and $P_{2}$ are not necessary disjoint, and possibly one of them consists of a single vertex, i.e., $u=u^{\prime}$ or $v=v^{\prime}$.

The pair of cycles $C$ and $C^{\prime}$ in Fig. 5(a) (resp., Fig. 5(b)) is a forbidden cycle pair, because there is a pair of a $u, u^{\prime}$-path $P_{1}=\left(u, x, z, y, u^{\prime}\right)$ and a $v, v^{\prime}$-path $P_{2}=\left(v, x^{\prime}, z, y^{\prime}, v^{\prime}\right)$ that satisfy the above conditions (i) (resp., (ii)). Note that the pair of cycles $C$ and $C^{\prime}$ in Fig. 2(a)-(b) is not forbidden cycle pair, because there are no such paths.

Our main result of this paper is as follows.

Theorem 1. A circular instance $(G, \gamma)$ admits an SLD cross-preserving embedding if and only if it has no forbidden cycle pair. Finding an SLD cross-preserving embedding of $\gamma$ or a forbidden cycle pair in $\mathcal{G}$ can be computed in linear time.

Proof of necessity: The necessity of the theorem follows from the next lemma.

For a cycle $C=(u, c, v, s) \in \mathcal{C}^{+}$(resp., $\mathcal{C}^{-}$) with $u, v \in V$ and $c, s \in \chi$ in $\mathcal{G}$, we call a vertex $z \in V$ an in-factor of $C$ if the exterior of $C \in \mathcal{C}^{+}$(resp., the interior of $\left.C \in \mathcal{C}^{-}\right)$has a $z, u$-path $P_{z, u}$ and a $z, v$-path $P_{z, v}$, i.e., $V\left(P_{z, u}-\right.$ $\{u\}) \cup V\left(P_{z, v}-\{v\}\right)$ is in $V_{\text {ex }}(C)$ (resp., $\left.V_{\text {in }}(C)\right)$. Paths $P_{z, u}$ and $P_{z, v}$ are not necessarily disjoint.

Lemma 4. Given $\mathcal{G}=\mathcal{G}(G, \gamma)$, let $\gamma^{\prime}$ be a cross-preserving embedding of $\gamma$. Then:

(i) Let $z \in V$ be an in-factor of a cycle $C \in \mathcal{C}^{+} \cup \mathcal{C}^{-}$in $\mathcal{G}$. Then cycle $C$ is a posi-cycle (resp., a nega-cycle) in $\mathcal{G}\left(G, \gamma^{\prime}\right)$ if and only if $z$ is in the exterior (resp., interior) of $C$ in $\gamma^{\prime}$; 


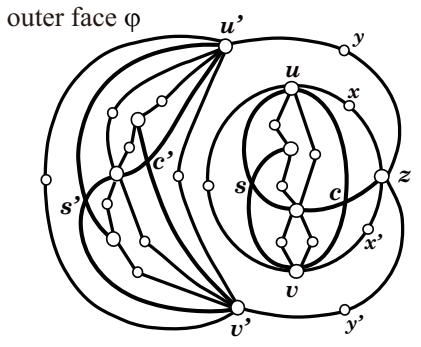

(a)

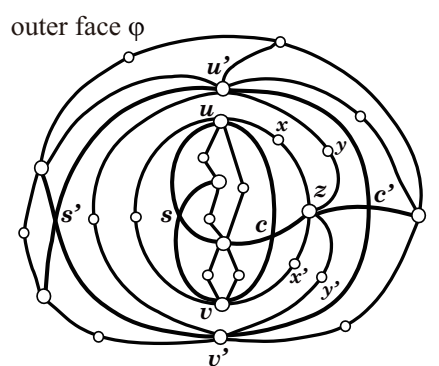

(b)

Fig. 5. Illustration of circular instances $(G, \gamma)$ with a cut-vertex $z$ of $\mathcal{G}$, where the crossing edges are depicted by slightly thicker lines: (a) forbidden cycle pair with hard B-cycles $C=(u, c, v, s)$ and $C^{\prime}=\left(u^{\prime}, c^{\prime}, v^{\prime}, s^{\prime}\right)$ (b) forbidden cycle pair with a hard B-cycle $C=(u, c, v, s)$ and a nega-cycle $C^{\prime}=\left(u^{\prime}, c^{\prime}, v^{\prime}, s^{\prime}\right)$ whose reversal is a hard B-cycle, where vertices $u, v, u^{\prime}, v^{\prime} \in V$ and crossings $c, s, c^{\prime}, s^{\prime} \in \chi$.

(ii) For a forbidden cycle pair $\left\{C, C^{\prime}\right\}$, one of $C$ and $C^{\prime}$ is a posi-cycle in $\mathcal{G}\left(G, \gamma^{\prime}\right)$ (hence any cross-preserving embedding of $\gamma$ contains a $B$ - or $W$-configuration and $(G, \gamma)$ admits no SLD cross-preserving embedding).

Proof of sufficiency: In the rest of paper, we prove the sufficiency of Theorem 1 by designing a linear-time algorithm that constructs an SLD crosspreserving embedding of an instance without a forbidden cycle pair.

\section{Biconnected Case}

In this section, $(G, \gamma)$ stands for a circular instance such that the vertex-connectivity of the plane graph $\mathcal{G}$ is at least 2 . In a biconnected graph $\mathcal{G}$, any two posi-cycles $C=(u, c, v, s), C^{\prime}=\left(u^{\prime}, c^{\prime}, v^{\prime}, s^{\prime}\right) \in \mathcal{C}^{+}$with $u, v, u^{\prime}, v^{\prime} \in V$ give a forbidden cycle pair if they do not share an inner face, because there is a pair of $u, u^{\prime}$-path and $v, v^{\prime}$-path in the exterior of $C$ and $C^{\prime}$. Analogously any pair of a posi-cycle $C$ and a nega-cycle $C^{\prime}$ such that $C^{\prime}$ encloses $C$ is also a forbidden cycle pair in a biconnected graph $\mathcal{G}$.

To detect such a forbidden pair in $\mathcal{G}$ in linear time, we first compute the sets $\mathcal{C}_{\mathrm{p}}, \mathcal{C}_{\mathrm{n}}, \mathcal{C}_{\mathrm{W}}, \mathcal{C}_{\mathrm{B}}, \mathcal{C}^{+}$and $\mathcal{C}^{-}$in $\gamma$ in linear time by using the inclusion-forest from Lemma 2.

Lemma 5. Given $(G, \gamma)$, the following in (i)-(iv) can be computed in $O(n)$ time.

(i) The sets $\mathcal{C}_{\mathrm{p}}, \mathcal{C}_{\mathrm{n}}$ and the inclusion-forest $\mathcal{I}$ of $\mathcal{C}_{\mathrm{p}} \cup \mathcal{C}_{\mathrm{n}} \cup\left\{C_{f} \mid f \in F(\gamma)\right\}$;

(ii) The sets $\mathcal{C}_{\mathrm{W}}$ and $\mathcal{C}_{\mathrm{B}}$;

(iii) The sets $\mathcal{C}^{+}, \mathcal{C}^{-}$and the inclusion-forest $\mathcal{I}^{*}$ of $\mathcal{C}^{+} \cup \mathcal{C}^{-}$; and

(iv) A set $\left\{f_{C} \mid C \in\left(\mathcal{C}_{\mathrm{W}} \cup \mathcal{C}_{\mathrm{B}}\right)-\mathcal{C}^{+}\right\}$such that $f_{C}$ is an inner face in the interior of a soft $B$ - or $W$-cycle $C$ with $V\left(C_{f}\right) \supseteq V(C)$. 
Given $(G, \gamma)$, a face $f \in F(\gamma)$ is called admissible if all posi-cycles enclose $f$ but no nega-cycle encloses $f$. Let $A(\gamma)$ denote the set of all admissible faces in $F(\gamma)$

Lemma 6. Given $(G, \gamma)$, it holds $A(\gamma) \neq \emptyset$ if and only if no forbidden cycle pair exists in $\gamma$. A forbidden cycle pair, if one exists, and $A(\gamma)$ can be obtained in $O(n)$ time.

By the lemma, if $(G, \gamma)$ has no forbidden cycle pair, i.e., $A(\gamma) \neq \emptyset$, then any new embedding obtained from $\gamma$ by changing the outer face with a face in $A(\gamma)$ is a cross-preserving embedding of $\gamma$ which has no hard B- or W-cycle.

\subsection{Eliminating Soft B- and W-cycles}

Suppose that we are given a circular instance $(G, \gamma)$ such that $\mathcal{G}$ is biconnected and $\mathcal{C}^{+}=\emptyset$. We now show how to eliminate all soft B- and W-cycles in $\mathcal{G}$ in linear time using the inclusion-forest from Lemma 2 and the spindles from Lemma 3.

Lemma 7. Given $(G, \gamma)$ with $\mathcal{C}^{+}=\emptyset$, there exists an SLD cross-preserving embedding $\gamma^{\prime}=\left(\chi, \rho^{\prime}, \varphi^{\prime}\right)$ of $\gamma$ such that $V\left(C_{\varphi^{\prime}}\right) \supseteq V\left(C_{\varphi}\right)$ for the facial cycle $C_{\varphi}\left(\right.$ resp., $\left.C_{\varphi^{\prime}}\right)$ of the outer face $\varphi\left(\right.$ resp., $\left.\varphi^{\prime}\right)$, which can be constructed in $O(n)$ time.

Given an instance $(G, \gamma)$ with a biconnected graph $\mathcal{G}$, we can test whether it has either a forbidden cycle pair or an admissible face by Lemmas 5 and 6 . In the former, it cannot have an SLD cross-preserving embedding by Lemma 4. In the latter, we can eliminate all hard B- and W-cycles by choosing an admissible face as a new outer face, and then eliminate all soft B- and W-cycles by a flipping procedure based on Lemma 7. All the above can be done in linear time.

To treat the case where the vertex-connectivity of $\mathcal{G}$ is 1 in the next section, we now characterize 1-plane embeddings that can have an SLD cross-preserving embedding such that a specified vertex appears along the outer boundary. For a vertex $z \in V$ in a graph $G$, we call a 1-plane embedding $\gamma$ of $G$ z-exposed if vertex $z$ appears along the outer boundary of $\gamma$. We call $(G, \gamma) z$-feasible if it admits a $z$-exposed SLD cross-preserving embedding $\gamma^{\prime}$ of $\gamma$.

Lemma 8. Given $(G, \gamma)$ such that $A(\gamma) \neq \emptyset$, let $z$ be a vertex in $V$. Then:

(i) The following conditions are equivalent:

(a) $\gamma$ admits no z-exposed SLD cross-preserving embedding;

(b) $A(\gamma)$ contains no face $f$ with $z \in V\left(C_{f}\right)$; and

(c) $\mathcal{G}$ has a posi- or nega-cycle $C$ to which $z$ is an in-factor;

(ii) A z-exposed SLD cross-preserving embedding or a posi- or nega-cycle $C$ to which $z$ is an in-factor can be computed in $O(n)$ time. 


\section{One-connected Case}

In this section, we prove the sufficiency of Theorem 1 by designing a linear-time algorithm claimed in the theorem. Given a circular instance $(G, \gamma)$, where $\mathcal{G}$ may be disconnected, obviously we only need to test each connected component of $\mathcal{G}$ separately to find a forbidden cycle pair. Thus we first consider a circular instance $(G, \gamma)$ such that the vertex-connectivity of $\mathcal{G}$ is 1 ; i.e., $\mathcal{G}$ is connected and has some cut-vertices.

A block $B$ of $\mathcal{G}$ is a maximal biconnected subgraph of $\mathcal{G}$. For a biconnected graph $\mathcal{G}$, we already know how to find a forbidden cycle pair or an SLD crosspreserving embedding from the previous section. For a trivial block $B$ with $|V(B)|=2$, there is nothing to do. If some block $B$ of $\mathcal{G}$ with $|V(B)| \geq 3$ contains a forbidden cycle pair, then $(G, \gamma)$ cannot admit any SLD cross-preserving embedding by Lemma 4 .

We now observe that $\mathcal{G}$ may contain a forbidden cycle pair even if no single block of $\mathcal{G}$ has a forbidden cycle pair.

Lemma 9. For a circular instance $(G, \gamma)$ such that the vertex-connectivity of $\mathcal{G}$ is 1 , let $B_{1}$ and $B_{2}$ be blocks of $\mathcal{G}$ and let $P_{1,2}$ be a $z_{1}, z_{2}$-path of $\mathcal{G}$ with the minimum number of edges, where $V\left(B_{i}\right) \cap V\left(P_{1,2}\right)=\left\{z_{i}\right\}$ for each $i=1$, 2. If $\left.\gamma\right|_{B_{i}}$ has a posi- or nega-cycle $C_{i}$ to which $z_{i}$ is an in-factor for each $i=1,2$, then $\left\{C_{1}, C_{2}\right\}$ is a forbidden cycle pair in $\mathcal{G}$.

For a linear-time implementation, we do not apply the lemma for all pairs of blocks in $\mathcal{B}$. A block of $\mathcal{G}$ is called a leaf block if it contains only one cut-vertex of $\mathcal{G}$, where we denote the cut-vertex in a leaf block $B$ by $v_{B}$. Without directly searching for a forbidden cycle pair in $\mathcal{G}$, we use the next lemma to reduce a given embedding by repeatedly removing leaf blocks.

Lemma 10. For a circular instance $(G, \gamma)$ such that the vertex-connectivity of $\mathcal{G}=\mathcal{G}(G, \gamma)$ is 1 and a leaf block $B$ of $\mathcal{G}$ such that $\left.\gamma\right|_{B}$ is $v_{B}$-feasible, let $H=$ $G-\left(V(B)-\left\{v_{B}\right\}\right)$ be the graph obtained by removing the vertices in $V(B)-\left\{v_{B}\right\}$. Then

(i) The instance $\left(H,\left.\gamma\right|_{H}\right)$ is circular; and

(ii) If $\left(H,\left.\gamma\right|_{H}\right)$ admits an SLD cross-preserving embedding $\gamma_{H}^{*}$, then an SLD cross-preserving embedding $\gamma^{*}$ of $\gamma$ can be obtained by placing a $v_{B}$-exposed $S L D$ cross-preserving embedding $\gamma_{B}^{*}$ of $\left.\gamma\right|_{B}$ within a space next to the cutvertex $v_{B}$ in $\gamma_{H}^{*}$.

Given a circular instance $(G, \gamma)$ such that $\mathcal{G}=\mathcal{G}(G, \gamma)$ is connected, an algorithm Algorithm Re-Embed-1-Plane for Theorem 1 is designed by the following three steps.

The first step tests whether $\mathcal{G}$ has a block $B$ such that $\left.\gamma\right|_{B}$ has a forbidden cycle pair, based on Lemma 8. If one exists, the algorithm outputs a forbidden cycle pair and halts.

After the first step, no block has a forbidden cycle pair. In the current circular instance $(G, \gamma)$, one of the following holds: 
(i) the number of blocks in $\mathcal{G}$ is at least two and there is at most one leaf block $B$ such that $\left.\gamma\right|_{B}$ is not $v_{B}$-feasible;

(ii) $\mathcal{G}$ has two leaf blocks $B$ and $B^{\prime}$ such that $\left.\gamma\right|_{B}$ is not $v_{B^{-}}$-feasible and $\left.\gamma\right|_{B^{\prime}}$ is not $v_{B^{\prime}}$-feasible; and

(iii) the number of blocks in $\mathcal{G}$ is at most one.

In (ii), $v_{B}$ is an in-factor of a cycle $C$ in $\left.\gamma\right|_{B}$ and $v_{B^{\prime}}$ is an in-factor of a cycle $C^{\prime}$ in $\left.\gamma\right|_{B^{\prime}}$ by Lemma 8 , and we obtain a forbidden cycle pair $\left\{C, C^{\prime}\right\}$ by Lemma 9. Otherwise if (i) holds, then we can remove all leaf blocks $B$ such that $\left.\gamma\right|_{B}$ is not $v_{B}$-feasible by Lemma 10 . The second step keeps removing all leaf blocks $B$ such that $\left.\gamma\right|_{B}$ is not $v_{B}$-feasible until (ii) or (iii) holds to the resulting embedding. If (i) occurs, then the algorithm outputs a forbidden cycle pair and halts.

When all the blocks of $\mathcal{G}$ can be removed successfully, say in an order of $B^{1}, B^{2}, \ldots, B^{m}$, the third step constructs an embedding with no B- or Wcycles by starting with such an SLD embedding of $B^{m}$ and by adding an SLD embedding of $B^{i}$ to the current embedding in the order of $i=m-1, m-2, \ldots, 1$. By Lemma 10, this results in an SLD cross-preserving embedding of the input instance $(G, \gamma)$.

Note that we can obtain an SLD cross-preserving embedding $\gamma_{H^{1}}^{*}$ of $\gamma$ in the third step when the first and second step did not find any forbidden cycle pair. Thus the algorithm finds either an SLD cross-preserving embedding of $\gamma$ or a forbidden cycle pair. This proves the sufficiency of Theorem 1.

By the time complexity result from Lemma 8 , we see that the algorithm can be implemented in linear time.

\section{References}

1. Auer, C., Bachmaier, C., Brandenburg, F. J. , Gleißner, A., Hanauer, K., Neuwirth D., Reislhuber J.: Outer 1-Planar Graphs, Algorithmica, 74(4), 1293-1320 (2016)

2. Di Battista G., Tamassia R.: On-line Planarity Testing, SIAM J. on Comput., 25(5), 956-997 (1996)

3. Eades, P., Hong S-H., Katoh N., Liotta, G. Schweitzer P., Suzuki Y.: A linear Time Algorithm for Testing Maximal 1-planarity of Graphs with a Rotation System, Theor. Comput. Sci., 513, 65-76 (2013)

4. Fabrici I., Madaras T.: The Structure of 1-planar Graphs, Discrete Mathematics, 307(7-8), 854-865 (2007)

5. Fáry I. : On straight line representations of planar Graphs, Acta Sci. Math. Szeged, 11, 229-233 (1948)

6. Grigoriev A., Bodlaender H.: Algorithms for Graphs embeddable with few crossings per edge, Algorithmica, 49(1), 1-11 (2007)

7. Hong S-H., Eades P., Katoh N., Liotta G., Schweitzer P., Suzuki Y.: A linear-time Algorithm for Testing Outer-1-planarity, Algorithmica, 72(4), 1033-1054 (2015)

8. Hong S-H., Eades P., Liotta G., Poon S.: Fary's Theorem for 1-planar Graphs, In: J. Gudmundsson, J., J. Mestre J., Viglas T. (eds.) COCOON 2012 LNCS, vol. 7434, pp. 335-346. Springer, Heidelberg (2013) 
9. Hong S-H, Nagamochi H.: Re-embedding a 1-Plane Graph into a Straight-line Drawing in Linear Time, Technical Report TR 2016-002, Department of Applied Mathematics and Physics, Kyoto University (2016)

10. Hopcroft J. E., Tarjan R. E.: Dividing a Graph into Triconnected Components, SIAM J. on Comput., 2, 135-158 (1973)

11. Korzhik V. P., Mohar B.: Minimal Obstructions for 1-immersions and Hardness of 1-planarity Testing, J. Graph Theory, 72(1), 30-71 (2013)

12. Pach J., Toth G.: Graphs Drawn with Few Crossings per Edge, Combinatorica, 17(3), 427-439 (1997)

13. Ringel G.: Ein Sechsfarbenproblem auf der Kugel, Abh. Math. Semin. Univ. Hamb., 29, 107-117 (1965)

14. Thomassen C.: Rectilinear Drawings of Graphs, J. Graph Theory, 10(3), 335-341 (1988) 